\title{
Joint DOA and Polarization Estimation for Unequal Power Sources
}

\author{
Qingyuan Fang, ${ }^{1}$ Yong Han, ${ }^{1}$ Ming Jin, ${ }^{1}$ and Wenyi Dong ${ }^{2}$ \\ ${ }^{1}$ Harbin Institute of Technology, School of Electronics and Information Engineering, Harbin 150001, China \\ ${ }^{2}$ Beijing Huahang Radio Measurement \& Research Institute, Beijing 100013, China \\ Correspondence should be addressed to Yong Han; han8662033@163.com
}

Received 1 October 2014; Accepted 15 January 2015

Academic Editor: Heng-Tung Hsu

Copyright (c) 2015 Qingyuan Fang et al. This is an open access article distributed under the Creative Commons Attribution License, which permits unrestricted use, distribution, and reproduction in any medium, provided the original work is properly cited.

\begin{abstract}
For most joint direction of arrival (DOA) and polarization estimation methods, the performances of proposed methods in dealing with unequal power sources are not discussed. However, sources with unequal powers apparently exist widely in actual applications. In this study, we propose a joint DOA and polarization estimation method for unequal power sources by utilizing the invariance property of noise subspace (IPNS) to the power of sources. This work extends the IPNS method to the dual polarized antenna array for joint DOA and polarization estimation. Moreover, we theoretically prove that the IPNS remains valid even when the sources are correlated. The computer simulations illustrate that the proposed method can effectively estimate the DOA and polarization parameters as the power difference between sources increases, as opposed to the polarimetric multiple signal classification (MUSIC) algorithm, which suffers from degradation in resolution probability. In addition, the performances of the proposed method are provided, as well the Cramer Rao Bound (CRB), which show approximate performance as the polarimetric MUSIC algorithm.
\end{abstract}

\section{Introduction}

Accurate direction of arrival (DOA) and polarimetric information serve a key function in radar and communication systems for target estimation, detection, and recognition and source localization [1-4]. Over the past three decades, joint DOA and polarization estimation methods have been developed on the basis of diversely polarized antenna arrays. However, most of these methods have not studied the case that the sources have unequal powers, which is common in practical applications; for example, the received powers from different transmitting antennas may vary because of the spatial distribution of users and the power allocation strategies in multiple-input multiple-output (MIMO) systems; the active jamming, possessing significantly higher power than the actual targets, is generally used to misguide the passive radar. To resolve the weak sources with the vicinity of strong sources (usually called interferences), various beamformers for interference suppression are proposed to maximize the signal of interest when the DOAs and polarimetric information of the interferences are predetermined [5-7]. However, in passive radar systems without prior information of jamming, we need to estimate the DOAs and polarization parameters of all sources (including the targets and the jamming) in the presence of power differences.

For joint DOA and polarization estimation, one class of methods based on the multiple signal classification (MUSIC) algorithm is the most representative [8-11]. The MUSIC algorithm performs the eigenvalue decomposition (EVD) of the covariance matrix with respect to the antenna array output to obtain the signal and noise subspace. The DOAs and polarization parameters of the sources are obtained by utilizing the orthogonal property of the noise subspace eigenvectors and the steering vectors. However, in the case of finite data samples, the polarimetric MUSIC method is insufficient and even invalid for dealing with adjacent sources with large power level differences. To date, only the issue of the DOA estimation for unequal power sources has been discussed in the literature. In [12] two models were developed for intermittent interference, and the corresponding CRB was derived to provide insights into the influence of the jammer parameters. In [13], the MUSIC-like spatial spectrum was realized via eigen-beamforming normalization to estimate the DOAs of the weak and strong sources. However, 
the performance of this method still depends on the orthogonal property among signal subspace eigenvectors; such property is seriously affected by the finite data samples when confronted with unequal power sources. In [14], DOA estimation was achieved after cochannel interference rejection through subarray beamforming. However, the resolution capability significantly degrades when the signal to interference plus noise ratio (SINR) declines. In MIMO systems, the unequal power reception that causes network capacity degradation is solved through optimal transmission power allocation [15]. Olfat and Nader-Esfahani presented a subspace method for DOA estimation on the basis of the invariance property of noise subspace (IPNS) to the power of sources; the method can effectively estimate the DOA of weak sources with the vicinity of strong sources [16]. The authors showed the capability of their method to handle the correlated sources in computer simulations. However, no proof was provided. The DOA estimation problem has been considered in all the aforementioned studies. However, the issue of joint DOA and polarization estimation for unequal power sources still requires further investigation.

In this study, we propose a joint DOA and polarization estimation method for unequal power sources by utilizing the IPNS to the power of sources. A general model for joint DOA and polarization estimation is derived on the basis of a dual polarized antenna array. The DOAs and polarization parameters of unequal power sources are obtained by exploiting the IPNS to the power of sources. However, in [16], the conclusion is derived according to the condition that the sources are independent. In the present study, we theoretically prove that the IPNS to the power of sources still holds when the sources are correlated. Computer simulations show that the proposed method has better resolution probability than the polarimetric MUSIC algorithm in dealing with adjacent unequal power sources. Even for sources with identical powers, the proposed method provides enhanced resolution probability. The performance analyses indicate that the proposed method is better than the polarimetric MUSIC algorithm in DOA estimations, despite not being as accurate as the latter in polarization estimations.

This paper is organized as follows. Section 2 discusses signal model based on the dual polarized antenna array for joint DOA and polarization estimation. Section 3 proposes a joint DOA and polarization estimation method using the IPNS to the power of sources. The method is proven effective for correlated sources. Section 4 provides the simulation results to evaluate the performance of the proposed method compared with that of the polarimetric MUSIC algorithm. Finally, Section 5 presents the conclusion.

\section{Data Model}

We consider a $2 M$-element uniform linear array (ULA) consisting of $M$ pairs of crossed dipoles placed along the $y$-axis with interelement spacing $d$, as shown in Figure 1. Suppose that $p$ narrow-band transverse electromagnetic (TEM) waves impinge on the ULA from the direction $\left(\theta_{i}, \varphi_{i}\right)$, $i=1,2, \ldots, P$. For simplicity, it is assumed that all the sources

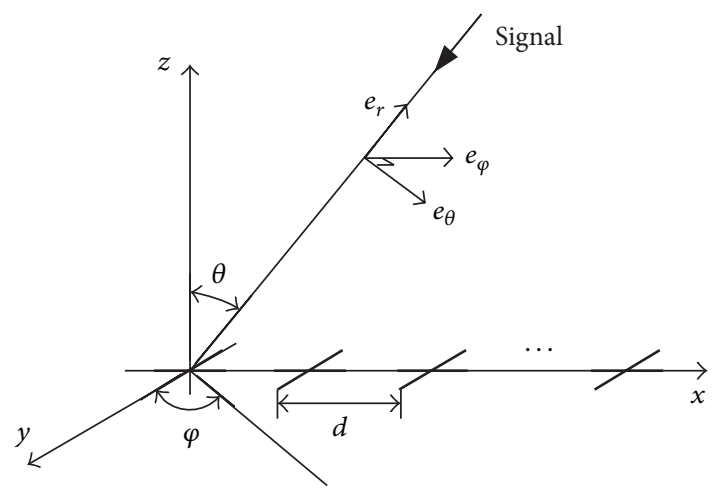

FIGURE 1: Uniform linear array configuration.

are in the $y z$-plane; that is, $\varphi_{i}=90^{\circ}, i=1,2, \ldots, P$. It can be seen from Figure 1 that the crossed dipoles can only receive the electric field components of the waves along the $x$ - and $y$-axes and have no response to the electric field component along the $z$-axis. The received data $\mathbf{z}(\mathbf{r}, t)$ from the crossed dipole located at $\mathbf{r}$ can be written as

$$
\begin{aligned}
\mathbf{z}(\mathbf{r}, t) & =E_{0} \mathbf{u}(\theta, \gamma, \eta) e^{j(w t-\mathbf{k} \cdot \mathbf{r}+\phi)}=\mathbf{u}(\theta, \gamma, \eta) s(t) e^{-\mathbf{k} \cdot \mathbf{r}}, \\
s(t) & =E_{0} e^{j(w t+\phi)}
\end{aligned}
$$

where $E_{0}$ is the wave amplitude, $\phi$ is the initial phase of the TEM wave, and $\mathbf{k}$ is the propagation vector. The $\mathbf{u}(\theta, \gamma, \eta)$ is given as $[17,18]$

$$
\mathbf{u}(\theta, \gamma, \eta)=\left[\begin{array}{ll}
-\cos \gamma & \cos \theta \sin \gamma e^{j \eta}
\end{array}\right]^{T}
$$

where $[\cdot]^{T}$ denotes the transpose, $\gamma(0 \leq \gamma \leq \pi / 2)$ denotes the polarization angle, and $\eta(0 \leq \eta \leq 2 \pi)$ represents the polarization phase difference. According to (1)-(2), the output of the $k$ th $(k=1,2, \ldots, M)$ crossed dipoles can be written as

$$
\mathbf{z}_{k}(t)=\sum_{i=1}^{P} \mathbf{u}\left(\theta_{i}, \gamma_{i}, \eta_{i}\right) s_{i}(t) q^{k-1}\left(\theta_{i}\right)+\mathbf{n}_{k}(t)
$$

where $q\left(\theta_{i}\right)$ is the space phase factor,

$$
q\left(\theta_{i}\right)=e^{j(2 \pi d / \lambda) \sin \theta_{i}}
$$

and $\mathbf{n}_{k}(t)$ denotes the received noise components at the $k$ th crossed dipoles

$$
\mathbf{n}_{k}(t)=\left[n_{x}(t), n_{y}(t)\right]^{T}
$$

For brevity, the output of the ULA can be written in a matrix form as

$$
\mathbf{Z}(t)=\mathbf{A S}(t)+\mathbf{N}(t)
$$


where $\mathbf{Z}(t), \mathbf{S}(t)$, and $\mathbf{N}(t)$ are the column vectors that contain the ULA output, sources, and noise, respectively; that is,

$$
\begin{gathered}
\mathbf{Z}(t)=\left[\begin{array}{c}
\mathbf{z}_{1}(t) \\
\mathbf{z}_{2}(t) \\
\vdots \\
\mathbf{z}_{M}(t)
\end{array}\right], \\
\mathbf{S}(t)=\left[\begin{array}{c}
s_{1}(t) \\
s_{2}(t) \\
\vdots \\
s_{P}(t)
\end{array}\right], \\
\mathbf{N}(t)=\left[\begin{array}{c}
\mathbf{n}_{1}(t) \\
\mathbf{n}_{2}(t) \\
\vdots \\
\mathbf{n}_{M}(t)
\end{array}\right] .
\end{gathered}
$$

A denotes the antenna array manifold matrix

$$
\mathbf{A}=\left[\mathbf{a}\left(\theta_{1}, \gamma_{1}, \eta_{1}\right) \mathbf{a}\left(\theta_{2}, \gamma_{2}, \eta_{2}\right) \ldots \mathbf{a}\left(\theta_{P}, \gamma_{P}, \eta_{P}\right)\right],
$$

whose column vector $\mathbf{a}\left(\theta_{i}, \gamma_{i}, \eta_{i}\right)$ is presented by [19]

$$
\begin{aligned}
\mathbf{a}\left(\theta_{i}, \gamma_{i}, \eta_{i}\right)= & {\left[\begin{array}{llll}
1 & q\left(\theta_{i}\right) & \cdots & q\left(\theta_{i}\right)^{M-1}
\end{array}\right]^{T} } \\
& \otimes \mathbf{u}\left(\theta_{i}, \gamma_{i}, \eta_{i}\right),
\end{aligned}
$$

where $\otimes$ represents the Kronecker product.

The problem addressed in this study is the joint estimation of the DOAs and polarization parameters of all sources, as denoted by $\Omega=\left\{\left(\theta_{i}, \gamma_{i}, \eta_{i}\right) \mid i=1,2, \ldots, P\right\}$, from a finite number of snapshots of $\mathbf{Z}(t)$ which are taken at times $t_{j}, j=1,2, \ldots, L$. For the unique parameters estimations, the following assumptions are made.

(1) The number of sources is predetermined and is less than the number of array elements; that is, $P<M$.

(2) The interelement spacing between each pair of crossed dipoles $d$ satisfies $d<\lambda / 2$ to avoid ambiguity problem of DOA and polarization estimation.

(3) The $P$ sources are incoherent (independent or correlated) zero-mean stationary processes. The covariance matrix of the sources is written as

$$
\mathbf{R}_{s}=E\left[\mathbf{S}(t) \mathbf{S}(t)^{H}\right]
$$

where $E[\cdot]$ denotes the expectation operator, $(\cdot)^{H}$ represents the conjugate transpose, and the rank of $\mathbf{R}_{s}$ is $P$.

(4) The additive noise components received at each crossed dipole, which are independent of the sources, are stationary zero-mean complex white Gaussian processes with the covariance matrix

$$
\mathbf{R}_{n}=E\left[\mathbf{N}(t) \mathbf{N}(t)^{H}\right]=\sigma_{n}^{2} \mathbf{I},
$$

where $\sigma_{n}^{2}$ is the noise power received at each dipole and $\mathbf{I}$ is an $2 M \times 2 M$ identity matrix.
According to the aforementioned assumptions, the covariance matrix of the antenna array output is expressed as

$$
\mathbf{R}=E\left[\mathbf{Z}(t) \mathbf{Z}(t)^{H}\right]=\mathbf{A R}_{s} \mathbf{A}^{H}+\sigma_{n}^{2} \mathbf{I} .
$$

In practical situations, $\mathbf{R}$ is unavailable and is usually replaced by a maximum likelihood estimate $\widehat{\mathbf{R}}$ that can be obtained as follows:

$$
\widehat{\mathbf{R}}=\frac{1}{L} \sum_{j=1}^{L} \mathbf{Z}\left(t_{j}\right) \mathbf{Z}\left(t_{j}\right)^{H}
$$

We will discuss the joint estimation of DOA and polarization of the sources with large power level differences on the basis of this sample covariance matrix.

\section{Joint DOA and Polarization Estimation for Unequal Power Sources}

3.1. Subspace Decomposition. The EVD of $\mathbf{R}$ yields

$$
\mathbf{R}=\mathbf{U} \boldsymbol{\Sigma} \mathbf{U}^{H}=\mathbf{U}_{S} \boldsymbol{\Sigma}_{S} \mathbf{U}_{S}^{H}+\mathbf{U}_{N} \boldsymbol{\Sigma}_{N} \mathbf{U}_{N}^{H},
$$

where $\mathbf{U} \in \mathbb{C}^{2 M \times 2 M}$ and $\boldsymbol{\Sigma} \in \mathbb{C}^{2 M \times 2 M}$ are the eigenvectors and eigenvalues of $\mathbf{R}$, which are arranged in decreasing order as [20]

$$
\lambda_{1} \geq \lambda_{2} \geq \cdots \geq \lambda_{P} \geq \lambda_{P+1}=\lambda_{P+2}=\cdots=\lambda_{2 M}=\sigma_{n}^{2} .
$$

$\Sigma_{S}=\operatorname{diag}\left(\lambda_{1}, \lambda_{2}, \ldots, \lambda_{P}\right)$ comprises the first $P$ dominant eigenvalues and $\Sigma_{N}=\operatorname{diag}\left(\lambda_{P+1}, \lambda_{P+2}, \ldots, \lambda_{2 M}\right)$ is composed of the remaining $2 M-P$ small eigenvalues, which are equal to $\sigma_{n}^{2}$. $\mathbf{U}_{S} \in \mathbb{C}^{2 M \times P}$ and $\mathbf{U}_{N} \in \mathbb{C}^{M \times(M-P)}$ are the eigenvectors corresponding to $\Sigma_{S}$ and $\Sigma_{N}$, respectively. The space spanned by $\mathbf{U}_{S}\left(\operatorname{span}\left(\mathbf{U}_{S}\right)\right)$ and that spanned by $\mathbf{U}_{N}$ are called the signal and noise subspaces, respectively, which are orthogonal $\operatorname{span}\left(\mathbf{U}_{S}\right) \perp \operatorname{span}\left(\mathbf{U}_{N}\right)$. Meanwhile, the space spanned by the array manifold matrix $\mathbf{A}(\operatorname{span}(\mathbf{A}))$ is equal to that spanned by $\mathbf{U}_{S}$; that is, $\operatorname{span}(\mathbf{A})=\operatorname{span}\left(\mathbf{U}_{S}\right)$ and $\operatorname{span}(\mathbf{A}) \perp \operatorname{span}\left(\mathbf{U}_{N}\right)$. The polarimetric MUSIC algorithm utilizes the orthogonality between the signal and noise subspace to estimate the DOA and polarization of sources. In most applications, the sources have different power levels especially for passive radar systems in which the jamming usually possesses a significantly higher power level than the actual targets. However, in the presence of power difference between sources, the performance of the polarimetric MUSIC algorithm degrades distinctly when the sample covariance matrix in (13) is used for estimation instead of the ideal covariance matrix being used for estimation.

3.2. Joint DOA and Polarization Estimation Based on IPNS to the Power of Sources. In [16], the noise subspace is certified to remain invariant to the powers of sources. This invariance indicates if the direction and polarization parameters remain fixed and only the power levels of sources are changed, the last $2 M-P$ eigenvalues of the ideal covariance matrix $\mathbf{R}$ and 
the corresponding eigenvectors will be the same as before. We utilize this property to estimate the DOA and polarization parameters of sources.

The power level changing of one exact source can be simulated by introducing an auxiliary signal (a virtual signal that does not really exist and is only used for mathematical realization) that has the same DOA and polarization parameters with that exact source. An auxiliary signal is considered to impinge on the array from direction $\theta^{\prime}$ with the polarization angle $\gamma^{\prime}$ and the polarization phase difference $\eta^{\prime}$. Here, the auxiliary signal is defined as independent from all the sources and noise. Thus, we define a new covariance matrix $\mathbf{D}$ on the basis of the array output covariance matrix $\mathbf{R}$ in (12):

$$
\mathbf{D} \triangleq \mathbf{R}+\rho_{a}^{2} \mathbf{a}\left(\theta^{\prime}, \gamma^{\prime}, \eta^{\prime}\right) \mathbf{a}\left(\theta^{\prime}, \gamma^{\prime}, \eta^{\prime}\right)^{H}
$$

where $\rho_{a}^{2}$ is a positive constant scalar that denotes the power of the auxiliary signal and $\mathbf{a}\left(\theta^{\prime}, \gamma^{\prime}, \eta^{\prime}\right)$ is the array response vector. Assuming that all the impinging sources (independent or correlated) belong to the set $\Omega=\left\{\left(\theta_{i}, \gamma_{i}, \eta_{i}\right) \mid 1 \leq i \leq P\right\}$, when the auxiliary signal coincides with one of the sources, that is, $\left(\theta^{\prime}, \gamma^{\prime}, \eta^{\prime}\right) \in \Omega$, the introduction of the auxiliary signal in (16) is equivalent to the power increasing of those exact sources. As a special case, if the sources are independent, such that $\mathbf{R}_{s}=E\left[\mathbf{S}(t) \mathbf{S}(t)^{H}\right]=\operatorname{diag}\left(\rho_{1}^{2}, \rho_{2}^{2}, \ldots, \rho_{P}^{2}\right)$, where $\rho_{i}^{2}$ denotes the power (variance) of the $i$ th source, the noise subspace eigenvalues remain constant to the powers of sources [16]. This conclusion indicates that the noise subspace eigenvalues of the new covariance matrix $\mathbf{D}$ are the same as those of $\mathbf{R}$ if the DOA of auxiliary signal coincides with the sources.

Furthermore, we extend this conclusion for the joint DOA and polarization estimation of unequal power sources based on a dual polarized array. Moreover the case of correlated sources is also discussed. We concisely rewrite the IPNS to power of sources as follows.

Theorem 1. If and only if the auxiliary signal coincides with one of the sources, that is, $\left(\theta^{\prime}, \gamma^{\prime}, \eta^{\prime}\right) \in \Omega$, the eigenvalues of noise subspace will remain invariant.

Proof. The auxiliary is assumed to coincide with one of the sources, that is, the $i$ th source, where $\left(\theta^{\prime}, \gamma^{\prime}, \eta^{\prime}\right)=\left(\theta_{i}, \gamma_{i}, \eta_{i}\right)$. According to (16), the matrix $\mathbf{D}$ becomes

$$
\mathbf{D}=\mathbf{R}+\rho_{a}^{2} \mathbf{a}\left(\theta_{i}, \gamma_{i}, \eta_{i}\right) \mathbf{a}\left(\theta_{i}, \gamma_{i}, \eta_{i}\right)^{H} .
$$

We define a selective vector $\mathbf{w}$ :

$$
\mathbf{w}=\left[\begin{array}{lll}
\mathbf{0}_{1 \times(i-1)} & \mathbf{1} & \mathbf{0}_{1 \times(2 M-i)}
\end{array}\right]^{T} .
$$

$\mathbf{R}$ in (17) is replaced with the expression in (12); we then obtain

$$
\begin{aligned}
\mathbf{D} & =\mathbf{A} \mathbf{R}_{S} \mathbf{A}^{H}+\sigma_{n}^{2} \mathbf{I}+\rho_{v}^{2} \mathbf{a}\left(\theta_{i}, \gamma_{i}, \eta_{i}\right) \mathbf{a}\left(\theta_{i}, \gamma_{i}, \eta_{i}\right)^{H} \\
& =\mathbf{A R}_{S} \mathbf{A}^{H}+\sigma_{n}^{2} \mathbf{I}+\rho_{v}^{2} \mathbf{A} \mathbf{w}_{i} \mathbf{w}_{i}^{T} \mathbf{A}^{H}=\mathbf{A} \widetilde{\mathbf{R}}_{S} \mathbf{A}^{H}+\sigma_{n}^{2} \mathbf{I}
\end{aligned}
$$

where

$$
\widetilde{\mathbf{R}}_{S}(m, n)= \begin{cases}\mathbf{R}_{S}(m, n)+\rho_{v}^{2} & m=n=i \\ \mathbf{R}_{S}(m, n) & \text { otherwise }\end{cases}
$$

From (12), (14), and (15), the noise powers (variance) evidently determine the noise subspace eigenvalues. According to (19) and (20), the auxiliary signal just affects the $i$ th element in the diagonal of $\mathbf{R}_{S}$ only and has no extra effect on the others. Even if the sources are correlated such that $\mathbf{R}_{s} \neq$ $\operatorname{diag}\left(\rho_{1}^{2}, \rho_{2}^{2}, \ldots, \rho_{P}^{2}\right)$, a comparison of (19) to (12) reveals that the auxiliary signal only affects the sources covariance matrix. Hence, the eigenvalue decomposition (EVD) of $\mathbf{D}$ yields the following:

$$
\mathbf{D}=\widetilde{\mathbf{U}} \widetilde{\mathbf{\Sigma}} \widetilde{\mathbf{U}}^{H}=\widetilde{\mathbf{U}}_{S} \widetilde{\Sigma}_{S} \widetilde{\mathbf{U}}_{S}^{H}+\widetilde{\mathbf{U}}_{N} \widetilde{\Sigma}_{N} \widetilde{\mathbf{U}}_{N}^{H},
$$

where the eigenvalues of $\mathbf{D}$ in decreasing order are

$$
\tilde{\lambda}_{1} \geq \tilde{\lambda}_{2} \geq \cdots \geq \tilde{\lambda}_{P} \geq \tilde{\lambda}_{P+1}=\tilde{\lambda}_{P+2}=\cdots=\tilde{\lambda}_{2 M}=\sigma_{n}^{2},
$$

and $\widetilde{\Sigma}_{S}=\operatorname{diag}\left(\widetilde{\lambda}_{1}, \widetilde{\lambda}_{2}, \ldots, \widetilde{\lambda}_{P}\right)$ and $\widetilde{\Sigma}_{N}=\operatorname{diag}\left(\widetilde{\lambda}_{P+1}, \widetilde{\lambda}_{P+2}, \ldots\right.$, $\left.\tilde{\lambda}_{2 M}\right)$. Based on (15) and (22), we get $\widetilde{\Sigma}_{N}=\Sigma_{N}$. The certification reveals that if the DOA and polarization parameters of the auxiliary signal coincide with those of the true sources, then the auxiliary signal has no influence on the noise subspace of the array output covariance matrix $\mathbf{R}$.

However, when the auxiliary signal is different with any one of the sources $\left(\theta^{\prime}, \gamma^{\prime}, \eta^{\prime}\right) \notin \Omega$, the expression in (19) becomes

$$
\begin{aligned}
\mathbf{D}= & {\left[\mathbf{A}, \mathbf{a}\left(\theta^{\prime}, \gamma^{\prime}, \eta^{\prime}\right)\right]\left[\begin{array}{cc}
\mathbf{R}_{S} & \mathbf{0} \\
\mathbf{0} & \rho_{a}^{2}
\end{array}\right]\left[\mathbf{A}, \mathbf{a}\left(\theta^{\prime}, \gamma^{\prime}, \eta^{\prime}\right)\right]^{H} } \\
& +\sigma_{n}^{2} \mathbf{I}=\mathbf{A}^{\prime} \mathbf{R}^{\prime}{ }_{D S} \mathbf{A}^{\prime H}+\sigma_{n}^{2} \mathbf{I} .
\end{aligned}
$$

Implicitly, the auxiliary signal affects the array manifold matrix and the sources of covariance matrix simultaneously. The eigenvalues of $\mathbf{D}$ in decreasing order are

$$
\begin{aligned}
\tilde{\lambda}_{1} & \geq \tilde{\lambda}_{2} \geq \cdots \geq \tilde{\lambda}_{P}+1 \geq \tilde{\lambda}_{P+2}=\tilde{\lambda}_{P+3}=\cdots=\tilde{\lambda}_{2 M} \\
& =\sigma_{n}^{2},
\end{aligned}
$$

where $\widetilde{\Sigma}_{S}=\operatorname{diag}\left(\widetilde{\lambda}_{1}, \tilde{\lambda}_{2}, \ldots, \widetilde{\lambda}_{P+1}\right)$ and $\widetilde{\Sigma}_{N}=\operatorname{diag}\left(\widetilde{\lambda}_{P+2}\right.$, $\left.\tilde{\lambda}_{P, \ldots, \tilde{\lambda}_{2 M}}\right) . \widetilde{\Sigma}_{N}$ is a $(2 M-P-1) \times(2 M-P-1)$ diagonal matrix which is different from the $\Sigma_{N}($ a $(2 M-P) \times(2 M-$ $P$ ) diagonal matrix). Thus, we conclude that $\widetilde{\Sigma}_{N} \neq \Sigma_{N}$ if $\left(\theta^{\prime}, \gamma^{\prime}, \eta^{\prime}\right) \notin \Omega$, which means the noise subspace of the array output covariance matrix will change when the DOA and polarization parameters of the auxiliary signal are different from those of the true sources. This ends the proof.

Thus we can utilize Theorem 1 to jointly estimate the DOA and polarization parameters of the sources. When the DOA and polarization parameters of the auxiliary signal coincide with those of the sources, the noise subspace eigenvalues of the new array output covariance matrix (which contains the auxiliary signal) remain invariant as before. However, the ideal array output covariance matrix $\mathbf{R}$ is not available in real applications and is usually replaced with the maximum likelihood estimate $\widehat{\mathbf{R}}$ based on a finite number of samples 


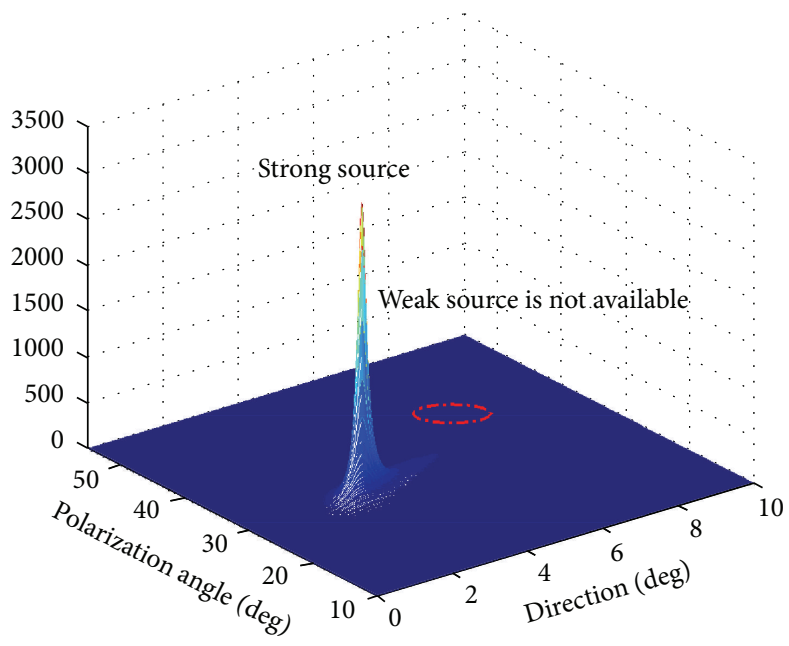

(a) The polarimetric MUSIC algorithm

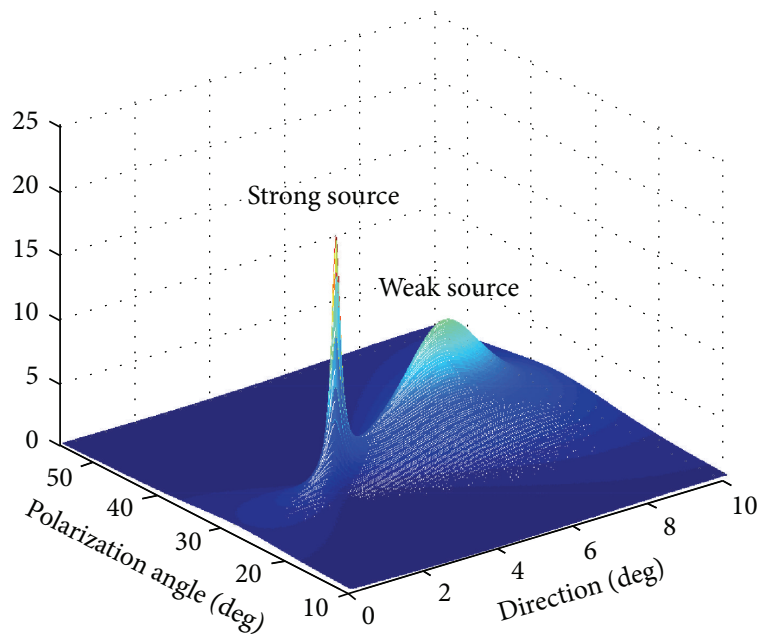

(b) The proposed method

FIGURE 2: Spectrums of the DOA and polarization estimation, where the strong source is located at $\left(\theta_{1}, \gamma_{1}\right)=\left(3^{\circ}, 30^{\circ}\right)$ SWR and the weak source is located at $\left(\theta_{2}, \gamma_{2}\right)=\left(7^{\circ}, 35^{\circ}\right)$.

in (13). Hence the noise subspace eigenvalues of the sample covariance matrix will not exactly satisfy

$$
\tilde{\lambda}_{i}=\lambda_{i}=\sigma_{n}^{2}, \quad i=P+1, P+2, \ldots, 2 M .
$$

Instead, we search for the sets of $\left(\theta^{\prime}, \gamma^{\prime}, \eta^{\prime}\right)$ that satisfy (25) as closely as possible instead. Thus, the procedures of the proposed algorithm are summarized as follows.

(1) The sample covariance matrix $\widehat{\mathbf{R}}$ in (13) is computed, and the eigenvalues of $\widehat{\mathbf{R}}$ are obtained, which in descending order are given by $\widehat{\lambda}_{1} \geq \widehat{\lambda}_{2} \geq \cdots \geq \hat{\lambda}_{2 M}$.

(2) The auxiliary signal is assumed to impinge on the antenna array from $\left(\theta^{\prime}, \gamma^{\prime}, \eta^{\prime}\right)$; then the new covariance matrix $\widehat{\mathbf{D}}$ in (17) is constructed, where the ideal covariance matrix $\mathbf{R}$ is replaced by $\widehat{\mathbf{R}}$. Let the positive constant scalar $\rho_{a}^{2}$ be equal to $\rho_{a}^{2}=\operatorname{tr}(\mathbf{R}) / 2 M(\operatorname{tr}(\cdot)$ denotes the matrix race) [16]; then the eigenvalues of $\widehat{\mathbf{D}}$ are obtained via EVD which are arranged in descending order as $\widehat{\tilde{\lambda}}_{1} \geq \widehat{\tilde{\lambda}}_{2} \geq \cdots \geq \widehat{\tilde{\lambda}}_{P} \geq$ $\widehat{\tilde{\lambda}}_{P+1} \geq \cdots \geq \widehat{\tilde{\lambda}}_{2 M}$.

(3) For each set of $\left(\theta^{\prime}, \gamma^{\prime}, \eta^{\prime}\right)$, the noise subspace eigenvalues of $\widehat{\mathbf{R}} \widehat{\lambda}_{i}, i=P+1, P+2, \ldots, 2 M$, are compared with those of $\widehat{\mathbf{D}} \widehat{\tilde{\lambda}}_{i}, i=P+1, P+2, \ldots, 2 M$, according to $G\left(\theta^{\prime}, \gamma^{\prime}, \eta^{\prime}\right)$, which is given as

$$
G\left(\theta^{\prime}, \gamma^{\prime}, \eta^{\prime}\right)=\frac{1}{\sum_{i=P+1}^{2 M}\left(\hat{\tilde{\lambda}}_{i}-\hat{\lambda}_{i}\right)} .
$$

(4) The joint estimation of the DOAs and polarization parameters of the actual sources comprises the $P$ sets of $\left(\theta^{\prime}, \gamma^{\prime}, \eta^{\prime}\right)$ that enable $G\left(\theta^{\prime}, \gamma^{\prime}, \eta^{\prime}\right)$ to reach $P$ maximums.

\section{Simulations}

A ULA that consists of $M=9$ pairs of crossed dipoles that are spaced half-wavelength apart is used to certify the performance of the proposed method. Two independent sources with unequal power are assumed to impinge on the ULA whose initial phases and polarization phase differences are both set at $\phi=0^{\circ}$ and $\eta=20^{\circ}$, respectively. We define the power of the strong source to that of the weak source ratio as the strong source to the weak source ratio (SWR), and the signal-to-noise ratio (SNR) is assumed as $10 \log _{10} \sigma^{2} \mathrm{~dB}$, where $\sigma^{2}$ denotes the signal power. The noise that is received by the crossed dipoles elements is an additive Gaussian process. Computer simulations with 200 independent Monte Carlo trials are conducted to assess the resolution ability and performance of the proposed method. In addition, the polarimetric MUSIC algorithm and the CRB are also applied for comparison [21].

4.1. Spectrums of the DOA and Polarization Estimation. In this simulation, we consider two unequal power sources that impinge on the antenna array with SWR $=20 \mathrm{~dB}$. The SNR of the weak source is $5 \mathrm{~dB}$ and a number of $L=200$ samples are employed. The spectrums that are obtained in one Monte Carlo trial through different methods are provided in Figure 2. Evidently, the two sources are estimated correctly by using the proposed method, as shown in Figure 2(b), whereas only the strong source is available when the polarimetric MUSIC algorithm is used, as shown in Figure 2(a). Hence, the resolution abilities are different for the different methods when a power difference exists between sources. The proposed method exhibits higher resolution capability for unequal power sources than the polarimetric MUSIC algorithm.

4.2. Resolution Probability. To see the resolution probability of the proposed method more clearly, we conduct several experiments to evaluate the effectiveness of estimating the weak sources in the presence of strong sources. In the following simulations, two unequal power sources are supposed to 


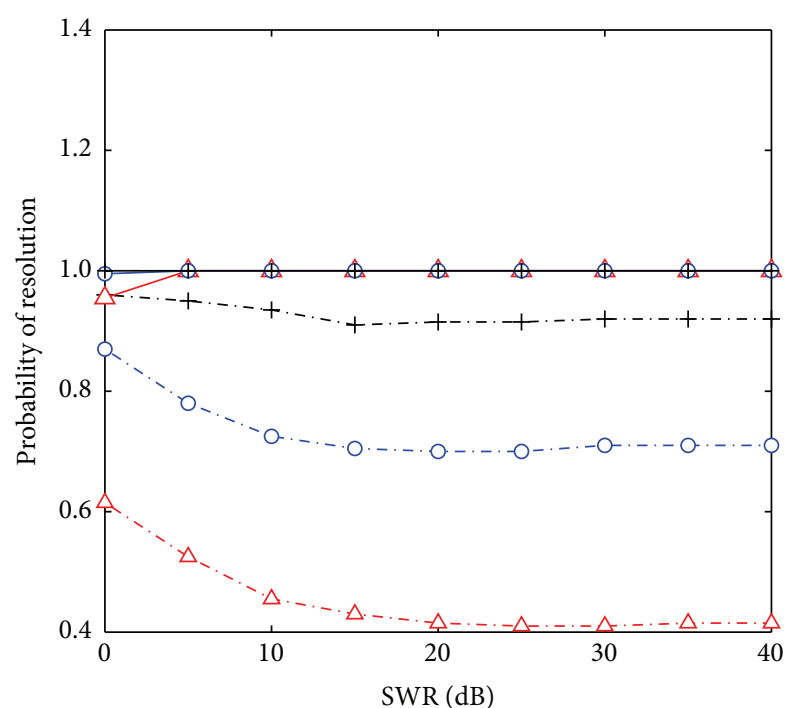

$$
\begin{array}{ll}
-\triangle-\text { MUSIC SNR }=5 \mathrm{~dB} & -\triangle \text { Proposed SNR }=5 \mathrm{~dB} \\
-\circ-\text { MUSIC SNR }=6 \mathrm{~dB} & \multimap-\text { Proposed SNR }=6 \mathrm{~dB} \\
--- \text { MUSIC SNR }=7 \mathrm{~dB} & \longrightarrow \text { Proposed SNR }=7 \mathrm{~dB}
\end{array}
$$

FIGURE 3: Resolution probability against the SWR, where the strong source is located at $\left(\theta_{1}, \gamma_{1}\right)=\left(3^{\circ}, 30^{\circ}\right)$ and the weak source is located at $\left(\theta_{2}, \gamma_{2}\right)=\left(7^{\circ}, 35^{\circ}\right)$.

impinge on the antenna array with a power difference. The resolution probabilities of the different methods are obtained for several scenarios to certify the resolution capability of the proposed method in resolving unequal power sources, where a number of $L=200$ samples are used in the following simulations. Suppose the directions $\widehat{\theta}_{1}, \widehat{\theta}_{2}$ are the DOA estimations for the two sources, whose true directions are $\theta_{1}, \theta_{2}$, so that one Monte Carlo trial is regarded as a successful estimation when the estimation results satisfy the following:

$$
\begin{aligned}
& \left|\widehat{\theta}_{1}-\theta_{1}\right| \leq \frac{\left|\theta_{1}-\theta_{2}\right|}{2} \\
& \left|\widehat{\theta}_{2}-\theta_{2}\right| \leq \frac{\left|\theta_{1}-\theta_{2}\right|}{2} .
\end{aligned}
$$

To see the influence of the power difference on the resolution probability, the SNR of the weak source is fixed as the SWR is increased. Figure 3 exhibits the resolution probabilities against the SWR, where the SWR varies over a range from $0 \mathrm{~dB}$ to $40 \mathrm{~dB}$. As shown in Figure 3 the resolution probability of the polarimetric MUSIC algorithm obviously suffers from degradation as the SWR increases, whereas the resolution probability of the proposed method increases as the SWR changes until the probability reaches $100 \%$. This finding means that the increasing SNR (power) of the strong source depresses the resolution probability of the polarimetric MUSIC algorithm but has minimal effects on the proposed method. In other words, the proposed method is more effective in resolving unequal power sources. Moreover, Figure 3 also shows that, despite the high resolution probability for unequal power sources of the proposed method, this method

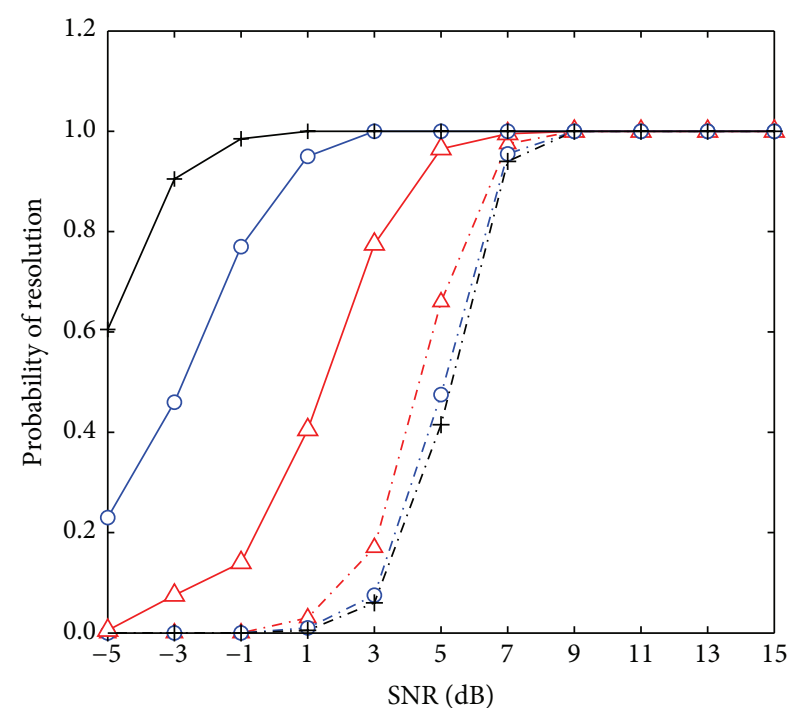

$$
\begin{array}{lll}
-\triangle-\text { MUSIC SWR }=0 \mathrm{~dB} & -\triangle & \text { Proposed SWR }=0 \mathrm{~dB} \\
-0-\text { MUSIC SWR }=10 \mathrm{~dB} & -0 \text { Proposed SWR }=10 \mathrm{~dB} \\
--- \text { MUSIC SWR }=20 \mathrm{~dB} & - \text { Proposed SWR }=20 \mathrm{~dB}
\end{array}
$$

FIGURE 4: Resolution probability against the SNR of the weak source, where the strong source is located at $\left(\theta_{1}, \gamma_{1}\right)=\left(3^{\circ}, 30^{\circ}\right)$ and the weak source is located at $\left(\theta_{2}, \gamma_{2}\right)=\left(7^{\circ}, 35^{\circ}\right)$ with SNR $=5 \mathrm{~dB}$.

also shows a higher resolution probability for sources with identical powers, as illustrated by $\mathrm{SWR}=0 \mathrm{~dB}$.

Figure 4 demonstrates the resolution probabilities against the SNR of the weak source, where the SNR varies over a range from $-5 \mathrm{~dB}$ to $15 \mathrm{~dB}$. For the low SNRs, the proposed method evidently shows a much higher resolution probability than the polarimetric MUSIC algorithm when confronted with unequal power sources. Noting that the proposed method is effective in dealing with unequal power sources, the resolution probability is enhanced as the power of the strong source increases, whereas the resolution probability deteriorates when the polarimetric MUSIC algorithm is used.

To examine the resolution probability for adjacent sources with a power difference, the direction and polarization angle of the strong source are fixed, whereas those of the weak source vary. Figures 5 and 6 indicate the resolution probabilities against the angular separation and polarimetric separation, respectively, where the corresponding angular separation varies over a range from 1 degree to 7 degrees and the polarimetric separation varies over a range from 10 degrees to 80 degrees. Figure 5 indicates that the proposed method has better resolution capability than polarimetric MUSIC algorithm for resolving adjacent sources with a power difference. The same conclusion is also derived from Figure 6.

4.3. Performance of the DOA and Polarization Estimation. To examine the performance of the proposed method, two unequal power sources are assumed to impinge on the antenna array with the $S W R=20 \mathrm{~dB}$, where the root mean square errors (RMSEs) are obtained as functions of the SNR with respect to the weak source. The RMSEs of the proposed 


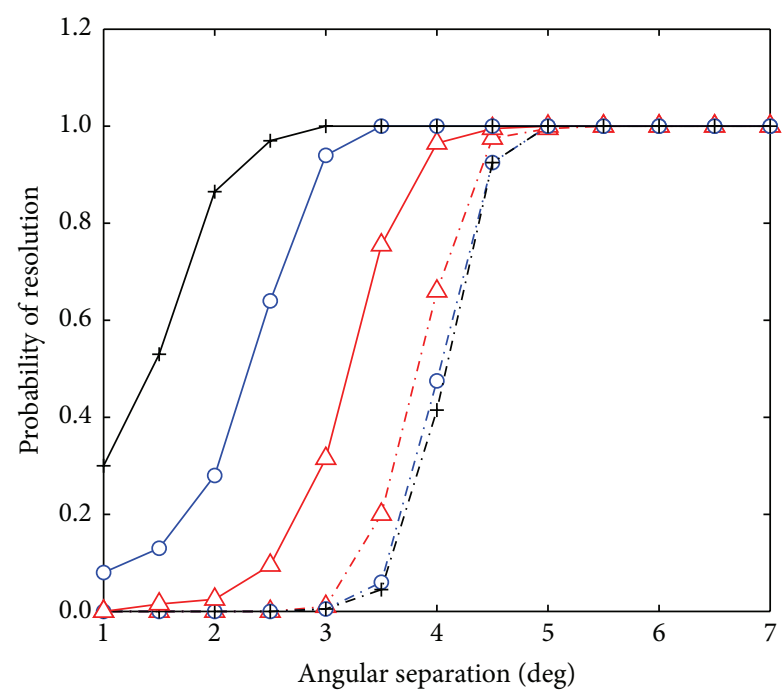

$$
\begin{array}{ll}
-\triangle-\text { MUSIC SWR }=0 \mathrm{~dB} & -\triangle \text { Proposed SWR }=0 \mathrm{~dB} \\
-0-\text { MUSIC SWR }=10 \mathrm{~dB} & -0 \text { Proposed SWR }=10 \mathrm{~dB} \\
--- & \text { MUSIC SWR }=20 \mathrm{~dB} \quad \longrightarrow \text { Proposed SWR }=20 \mathrm{~dB}
\end{array}
$$

FIGURE 5: Resolution probability against the angular separation, where the strong source is located at $\left(\theta_{1}, \gamma_{1}\right)=\left(3^{\circ}, 30^{\circ}\right)$ and the weak source is located at $\left(\theta_{2}, \gamma_{2}\right)=\left(\theta_{1}+\Delta, 35^{\circ}\right)$ with $\mathrm{SNR}=5 \mathrm{~dB}$.

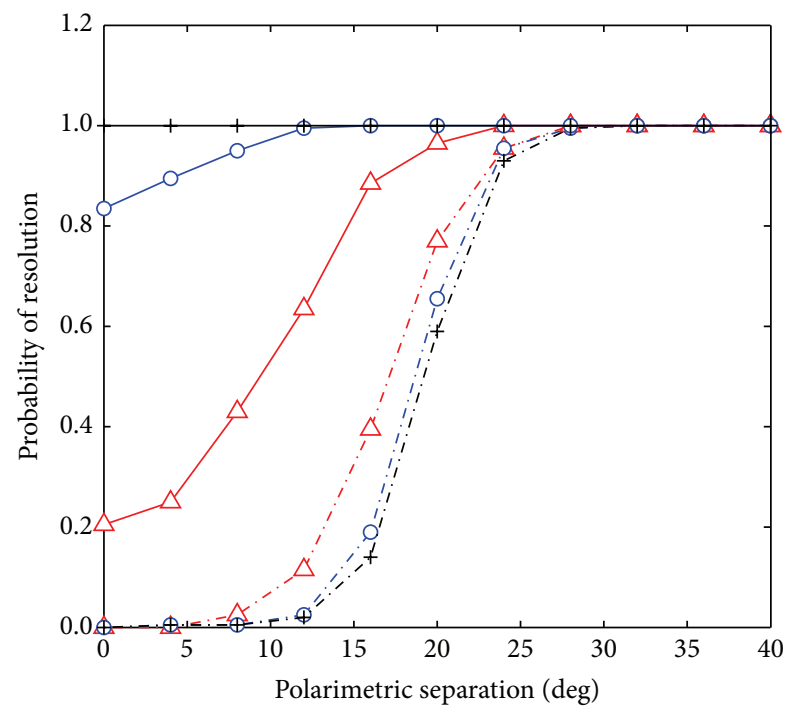

$$
\begin{aligned}
& \triangle-\text { MUSIC SWR }=0 \mathrm{~dB} \quad \triangle-\text { Proposed SWR }=0 \mathrm{~dB} \\
& -\circ-\text { MUSIC SWR }=10 \mathrm{~dB} \quad-0 \text { Proposed SWR }=10 \mathrm{~dB} \\
& -1-\text { MUSIC SWR }=20 \mathrm{~dB} \rightarrow \text { Proposed SWR }=20 \mathrm{~dB}
\end{aligned}
$$

FIGURE 6: Resolution probability against the polarimetric separation, where the strong source is located at $\left(\theta_{1}, \gamma_{1}\right)=\left(3^{\circ}, 30^{\circ}\right)$ and the weak source is located at $\left(\theta_{2}, \gamma_{2}\right)=\left(6^{\circ}, \gamma_{1}+\Delta\right)$ with SNR $=5 \mathrm{~dB}$.

method are compared with the polarimetric MUSIC algorithm and the CRB. Figures 7 and 8 exhibit the RMSEs of the DOA for the strong and weak sources, respectively. As shown in Figures 7 and 8, the RMSEs of the DOA for the strong source are almost the same with the two methods, and

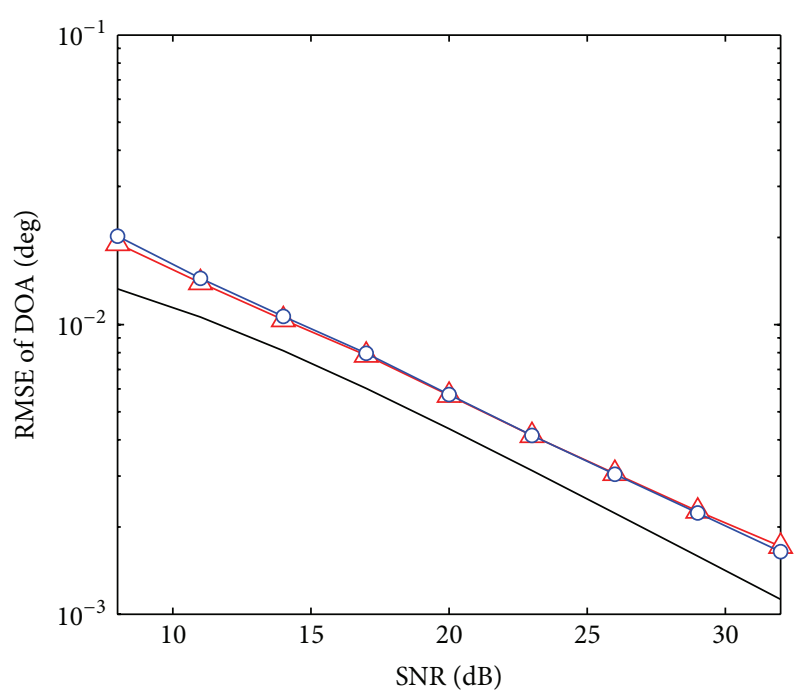

$$
\begin{aligned}
& \triangle \text { MUSIC } \\
& \multimap-\text { Proposed method } \\
& \longleftarrow \text { CRB }
\end{aligned}
$$

FIGURE 7: RMSE of the DOA for the strong source versus the SNR of the weak source, where the strong source is located at $\left(\theta_{1}, \gamma_{1}\right)=\left(3^{\circ}, 30^{\circ}\right)$ with SWR $=20 \mathrm{~dB}$ and the weak source is located at $\left(\theta_{2}, \gamma_{2}\right)=\left(7^{\circ}, 35^{\circ}\right)$.

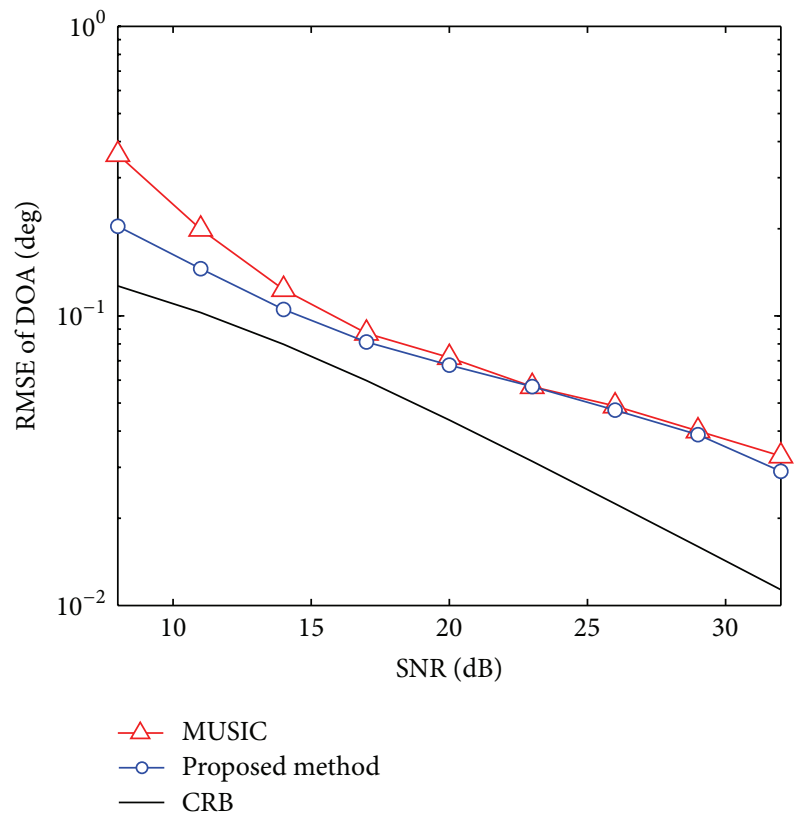

FIGURE 8: RMSE of the DOA for the weak source versus the SNR of the weak source, where the strong source is located at $\left(\theta_{1}, \gamma_{1}\right)=$ $\left(3^{\circ}, 30^{\circ}\right)$ with SWR $=20 \mathrm{~dB}$ and the weak source is located at $\left(\theta_{2}, \gamma_{2}\right)=\left(7^{\circ}, 35^{\circ}\right)$.

the RMSEs of the proposed method perform better for the weak source than the polarimetric MUSIC algorithm.

Figures 9 and 10 illustrate the RMSEs of the polarization angle for the strong and weak sources, respectively. As shown in Figures 9 and 10, despite its high resolution probability of the proposed method, this method is not as accurate 


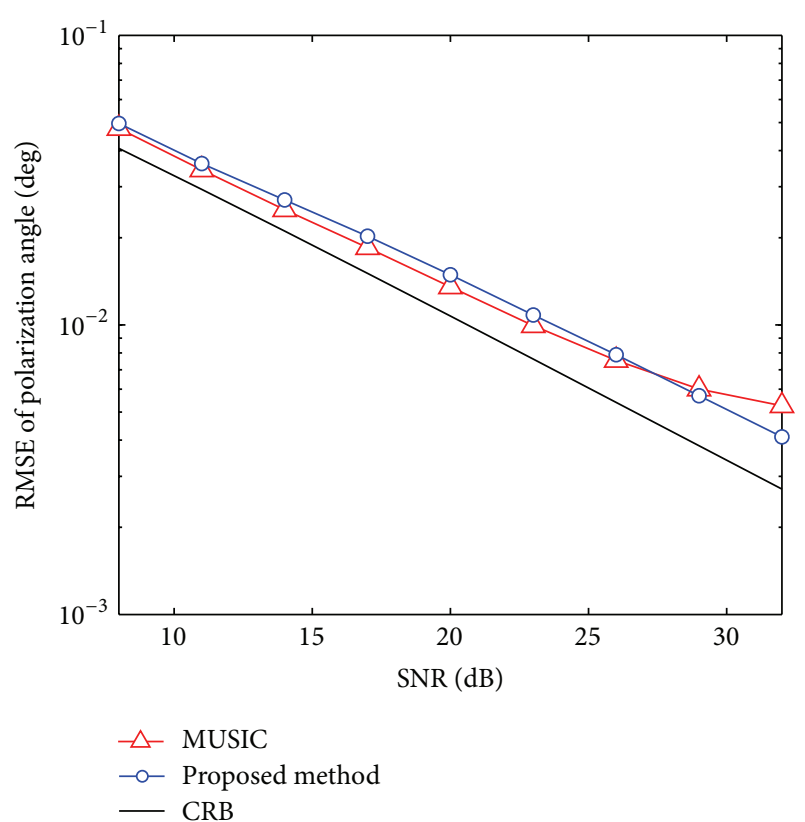

FIGURE 9: RMSE of the polarization angle for the strong source versus the SNR of the weak source, where the strong source is located at $\left(\theta_{1}, \gamma_{1}\right)=\left(3^{\circ}, 30^{\circ}\right)$ with SWR $=20 \mathrm{~dB}$ and the weak source is located at $\left(\theta_{2}, \gamma_{2}\right)=\left(7^{\circ}, 35^{\circ}\right)$.

as the polarimetric MUSIC algorithm in polarization angle estimation.

\section{Conclusion}

In this study, the IPNS to the power of sources is used to estimate the DOA and polarization for unequal power sources jointly on the basis of a dual polarized antenna array. We also theoretically proved that the proposed method is valid for both independent sources and correlated sources. As shown in the simulation results, the resolution probability of the proposed method continuously increases as the power difference between the sources increases, whereas the resolution probability degrades for the polarimetric MUSIC algorithm. Meanwhile, the proposed method also shows a much higher resolution probability than the polarimetric MUSIC algorithm when confronted with equal power sources. In addition, the proposed method has better performance than the polarimetric MUSIC algorithm in the DOA estimations, despite the fact that the proposed method is not as accurate as the polarimetric MUSIC algorithm in the polarization estimations. Compared with the polarimetric MUSIC algorithm, the proposed method needs an EVD in each direction when searching for the spatial and polarimetric spectrum peaks.

\section{Conflict of Interests}

The authors declare that there is no conflict of interests regarding the publication of this paper.

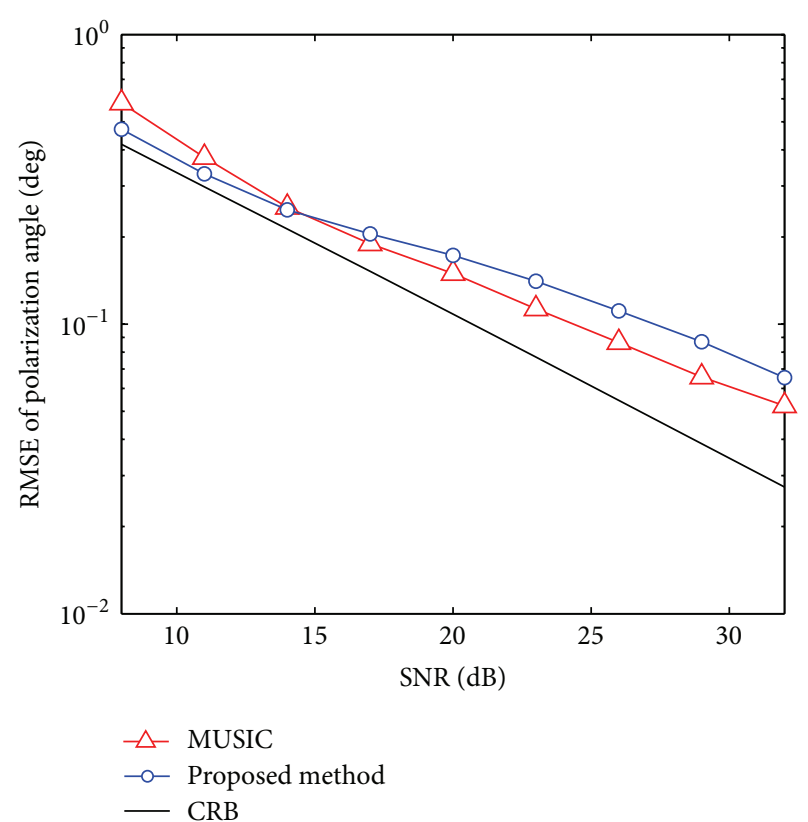

FIGURE 10: RMSE of the polarization angle for the weak source versus the SNR of the weak source, where the strong source is located at $\left(\theta_{1}, \gamma_{1}\right)=\left(3^{\circ}, 30^{\circ}\right)$ with SWR $=20 \mathrm{~dB}$ and the weak source is located at $\left(\theta_{2}, \gamma_{2}\right)=\left(7^{\circ}, 35^{\circ}\right)$.

\section{Acknowledgments}

This work was supported by the Natural Science Foundation of China (Grant no. 61271118) and Natural Scientific Research Innovation Foundation in Harbin Institute of Technology (HIT.NSRIF 2013130 and HIT(WH)XBQD 201022).

\section{References}

[1] A. Marino, S. R. Cloude, and I. H. Woodhouse, "A polarimetric target detector using the Huynen fork," IEEE Transactions on Geoscience and Remote Sensing, vol. 48, no. 5, pp. 2357-2366, 2010.

[2] J.-W. Tao, L. Liu, and Z.-Y. Lin, "Joint DOA, range, and polarization estimation in the fresnel region," IEEE Transactions on Aerospace and Electronic Systems, vol. 47, no. 4, pp. 26572672, 2011.

[3] J.-J. Xiao and A. Nehorai, "Joint transmitter and receiver polarization optimization for scattering estimation in clutter," IEEE Transactions on Signal Processing, vol. 57, no. 10, pp. 41424147, 2009.

[4] D. Rahamim, J. Tabrikian, and R. Shavit, "Source localization using vector sensor array in a multipath environment," IEEE Transactions on Signal Processing, vol. 52, no. 11, pp. 3096-3103, 2004.

[5] S. Daneshmand, A. Broumandan, J. Nielsen, and G. Lachapelle, "Interference and multipath mitigation utilising a two-stage beamformer for global navigation satellite systems applications," IET Radar, Sonar and Navigation, vol. 7, no. 1, pp. 55-66, 2013.

[6] R. Levanda and A. Leshem, "Adaptive selective sidelobe canceller beamformer with applications to interference mitigation 
in radio astronomy," IEEE Transactions on Signal Processing, vol. 61, no. 20, pp. 5063-5074, 2013.

[7] J.-W. Tao, "Performance analysis for interference and noise canceller based on hypercomplex and spatiotemporal-polarisation processes," IET Radar, Sonar and Navigation, vol. 7, no. 3, pp. 277-286, 2013.

[8] Y. Zhang, B. A. Obeidat, and M. G. Amin, "Spatial polarimetric time-frequency distributions for direction-of-arrival estimations," IEEE Transactions on Signal Processing, vol. 54, no. 4, pp. 1327-1340, 2006.

[9] P. Chevalier, A. Ferrol, L. Albera, and G. Birot, "Higher order direction finding from arrays with diversely polarized antennas: the PD-2q-MUSIC algorithms," IEEE Transactions on Signal Processing, vol. 55, no. 11, pp. 5337-5350, 2007.

[10] Y. Wu, H. C. So, C. Hou, and J. Li, "Passive localization of near-field sources with a polarization sensitive array," IEEE Transactions on Antennas and Propagation, vol. 55, no. 8, pp. 2402-2408, 2007.

[11] X. Yuan, "Estimating the DOA and the polarization of a polynomial-phase signal using a single polarized vector-sensor," IEEE Transactions on Signal Processing, vol. 60, no. 3, pp. 12701282, 2012.

[12] O. Besson, P. Stoica, and Y. Kamiya, "Direction finding in the presence of an intermittent interference," IEEE Transactions on Signal Processing, vol. 50, no. 7, pp. 1554-1564, 2002.

[13] L. Xu, C. Zeng, G.-S. Liao, and J. Li, "DOA estimation for strong and weak signals in the presence of array gain and phase mismatch," in Proceedings of the International Conference on Multimedia Technology (ICMT '10), pp. 1-4, Ningbo, China, October 2010.

[14] N. Wang, P. Agathoklis, and A. Antoniou, "A new DOA estimation technique based on subarray beamforming," IEEE Transactions on Signal Processing, vol. 54, no. 9, pp. 3279-3289, 2006.

[15] A. Zanella, M. Chiani, and M. Z. Win, "The effect of unequal power reception in cellular MIMO networks," Signal Processing, vol. 90, no. 6, pp. 1850-1860, 2010.

[16] A. Olfat and S. Nader-Esfahani, "A new signal subspace processing for DOA estimation," Signal Processing, vol. 84, no. 4, pp. 721-728, 2004.

[17] J. Li and R. T. Compton Jr., "Angle and polarization estimation using ESPRIT with a polarization sensitive array," IEEE Transactions on Antennas and Propagation, vol. 39, no. 9, pp. 1376-1383, 1991.

[18] J. Li and R. T. Compton Jr., "Angle estimation using a polarization sensitive array," IEEE Transactions on Antennas and Propagation, vol. 39, no. 10, pp. 1539-1543, 1991.

[19] Y. Hua, "A pencil-MUSIC algorithm for finding two-dimensional angles and polarizations using crossed dipoles," IEEE Transactions on Antennas and Propagation, vol. 41, no. 3, pp. 370-376, 1993.

[20] R. O. Schmidt, "Multiple emitter loacation and signal parameter estimation," IEEE Transactions on Antennas and Propagation, vol. 34, no. 3, pp. 276-280, 1986.

[21] A. J. Weiss and B. Friedlander, "Performance analysis of diversely polarized antenna arrays," IEEE Transactions on Signal Processing, vol. 39, no. 7, pp. 1589-1603, 1991. 

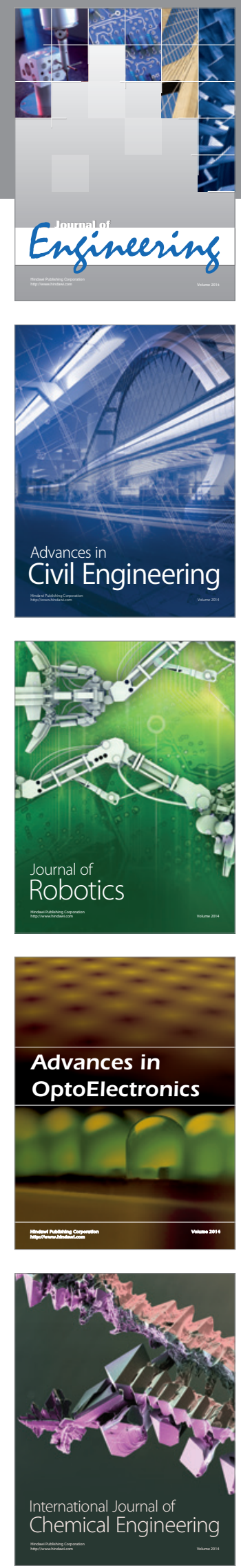

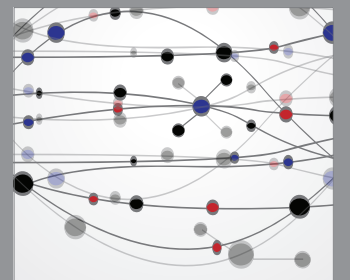

The Scientific World Journal
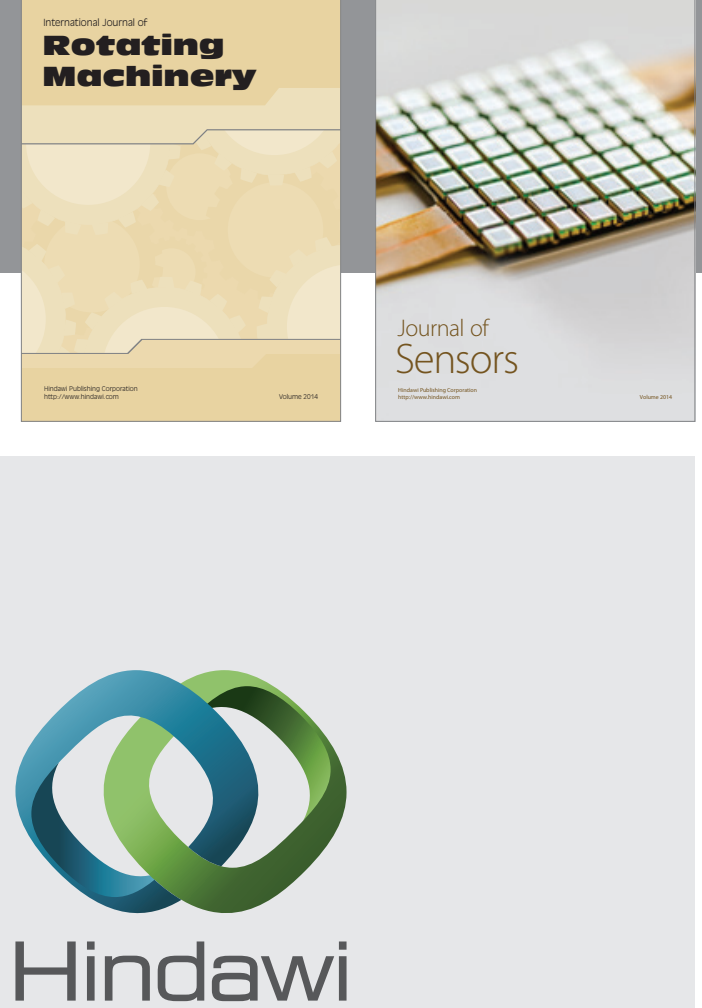

Submit your manuscripts at http://www.hindawi.com
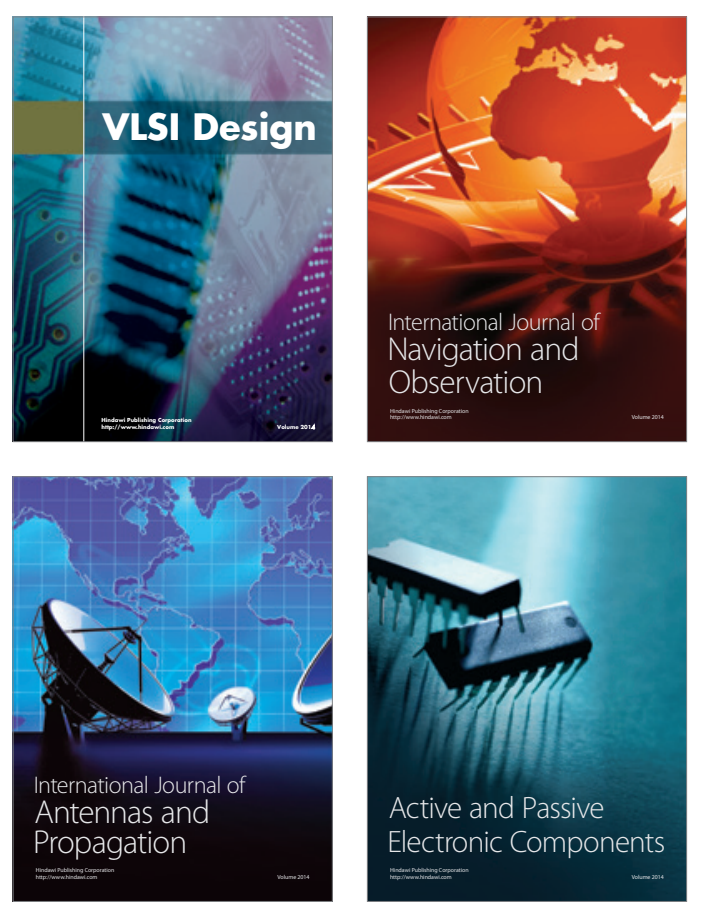
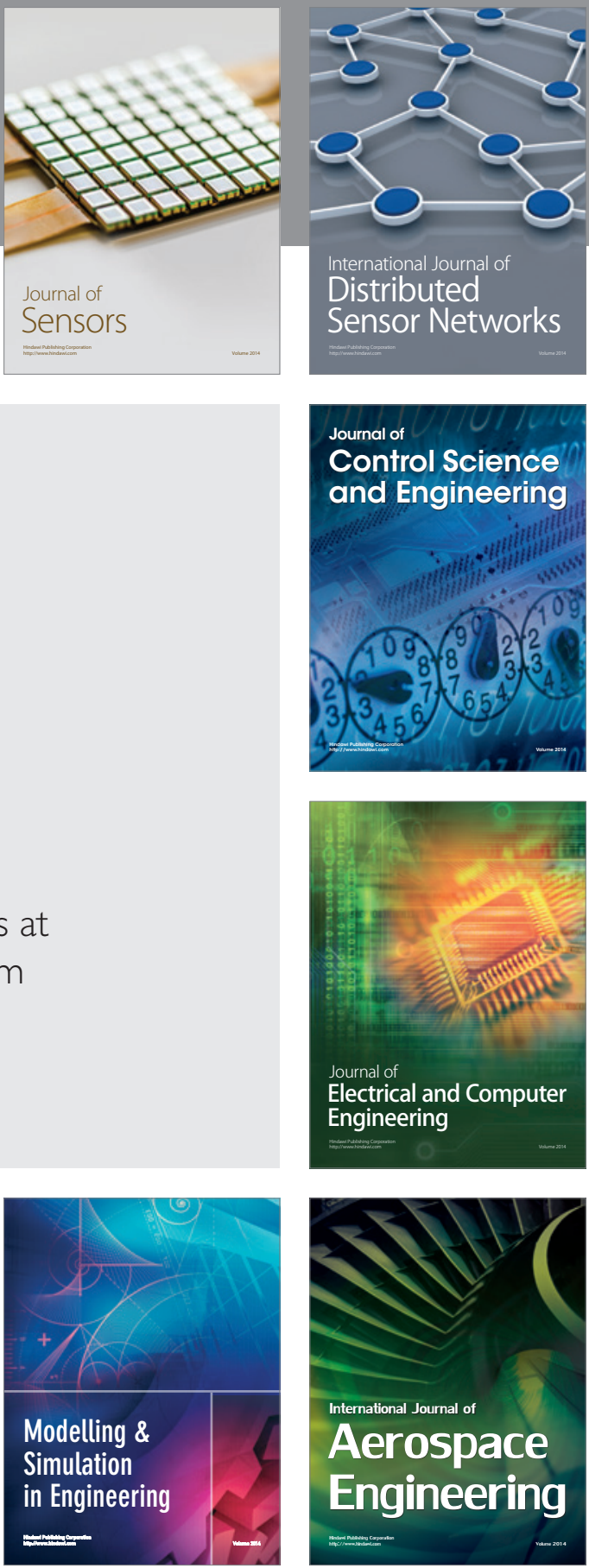

Journal of

Control Science

and Engineering
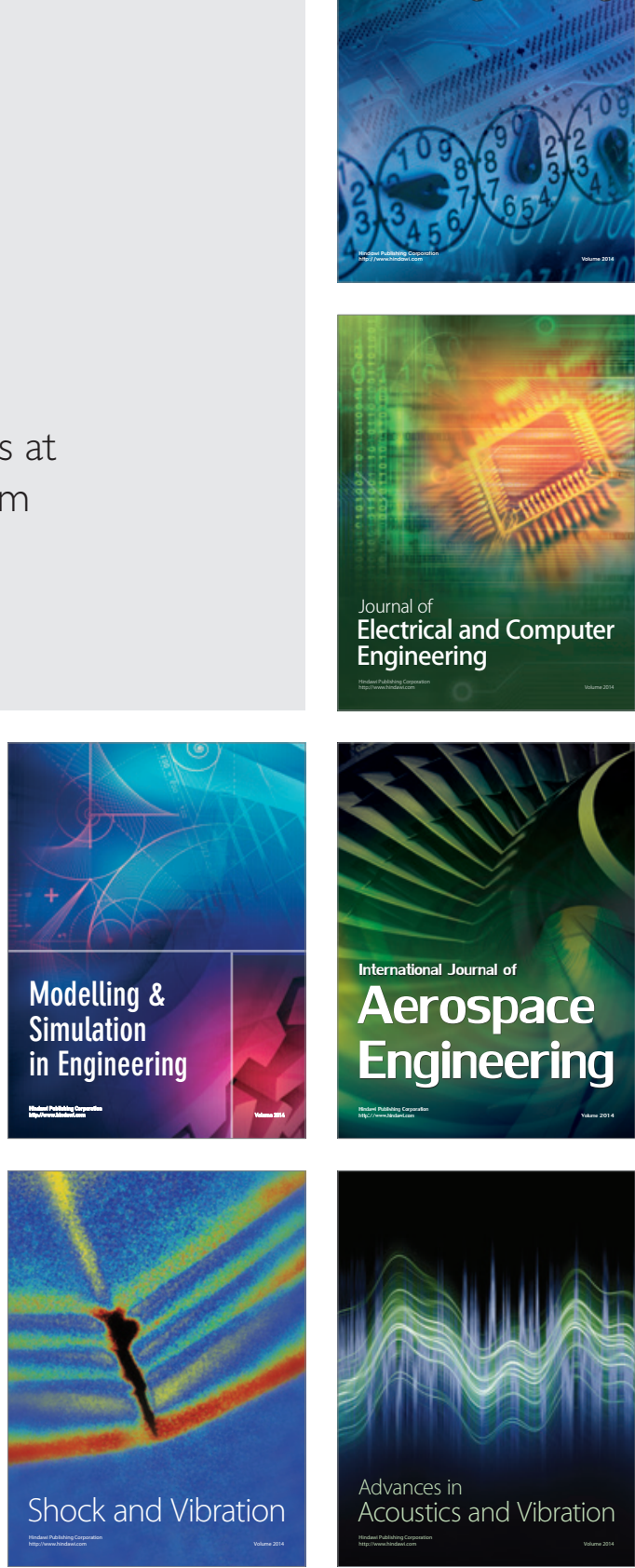